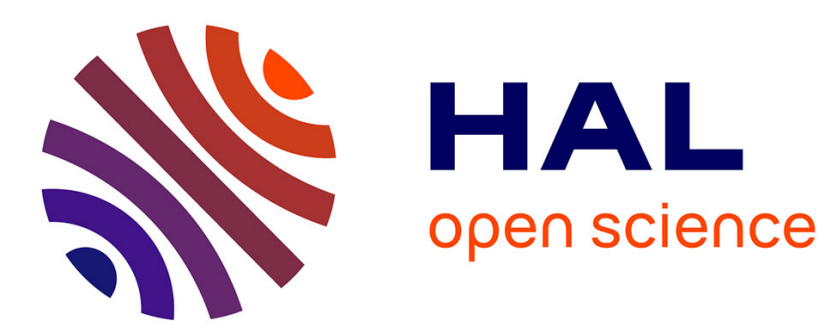

\title{
Traitement d'images astronomiques dégradées par des fluctuations atmosphériques
}

F. Roddier

\section{To cite this version:}

F. Roddier. Traitement d'images astronomiques dégradées par des fluctuations atmosphériques. Revue de Physique Appliquée, 1976, 11 (2), pp.195-201. 10.1051/rphysap:01976001102019500 . jpa00244049

\section{HAL Id: jpa-00244049 https://hal.science/jpa-00244049}

Submitted on 1 Jan 1976

HAL is a multi-disciplinary open access archive for the deposit and dissemination of scientific research documents, whether they are published or not. The documents may come from teaching and research institutions in France or abroad, or from public or private research centers.
L'archive ouverte pluridisciplinaire HAL, est destinée au dépôt et à la diffusion de documents scientifiques de niveau recherche, publiés ou non, émanant des établissements d'enseignement et de recherche français ou étrangers, des laboratoires publics ou privés. 


\section{TRAITEMENT D'IMAGES ASTRONOMIQUES DÉGRADÉES PAR DES FLUCTUATIONS ATMOSPHÉRIQUES}

\section{F. RODDIER}

Département d'Astrophysique, I. M. S. P., Université de Nice, 06034 Nice-Cedex, France

Résumé. - Après avoir passé en revue les théories actuelles sur la formation des images astronomiques à travers la turbulence atmosphérique, on décrit diverses méthodes employées pour restaurer l'information : interférométrie, speckle-interférométrie, déconvolution. Une restauration complète de l'information est théoriquement possible par analyse statistique d'un grand nombre d'images instantanées.

La turbulence atmosphérique affecte considérablement la qualité des images astronomiques. La figure 1 montre une série de clichés de l'étoile double Castor obtenus par J. Rösch à l'Observatoire du Pic du Midi. La distance entre les deux composantes est de 2 secondes

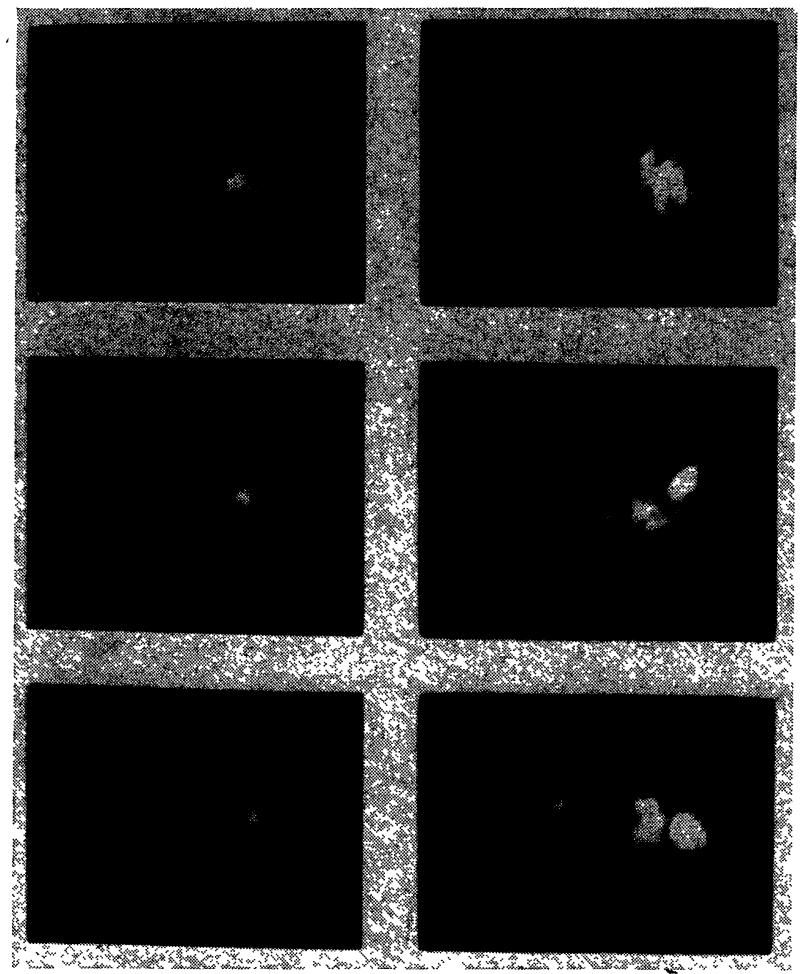

FIG. 1. - Photographies à très court temps de pose de l'étoile double Castor (Cliché J. Rösch, Observatoire du Pic du Midi).

d'arc. L'aspect de l'image varie rapidement au cours du temps, de sorte que des temps de pose de l'ordre de quelques millisecondes sont nécessaires pour figer ainsi l'aspect instantané de l'image.

On remarque sur ces clichés la similitude entre les déformations subies par chacune des deux composantes de l'étoile double. Cela se produit lorsque les faisceaux lumineux issus de chacune des deux étoiles traversent les mêmes régions turbulentes de l'atmosphère. Les aberrations introduites sont alors isoplanétiques, c'est-à-dire indépendantes de la direction d'observation. On connaît mal l'étendue du domaine d'isoplanétisme, mais on admet que pour des objets de petite dimension angulaire, cette condition est vérifiée (Fig. 2). La relation entre la répartition $O(\alpha)$ des

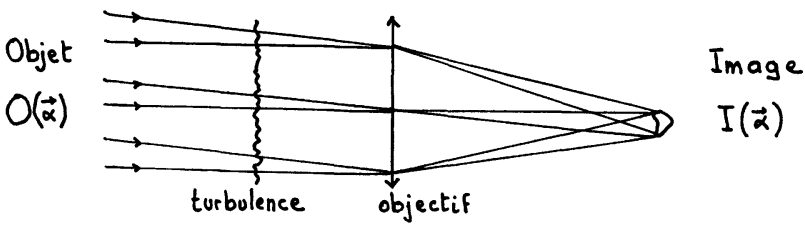

Fig. 2. - Lorsque l'objet observé a des dimensions angulaires suffisamment petites ou lorsque la turbulence est localisée au voisinage de la pupille du télescope, les faisceaux issus des différents points de l'objet traversent les mêmes régions turbulentes et les aberrations introduites sont isoplanétiques.

brillances dans l'objet (incohérent) et la répartition instantanée $I(\alpha)$ des éclairements dans l'image est alors une relation de convolution $[1,2,3]$

$$
I(\alpha)=O(\alpha) * S(\alpha) .
$$

Dans cette expression $I(\alpha)$ et $S(\alpha)$ sont aléatoires. $S(\alpha)$ représente la répartition instantanée des éclairements que produirait une source lumineuse ponctuelle (étoile non résolue) en fonction de la direction d'observation $\alpha$ sur le ciel. C'est un ensemble de taches lumineuses comparées par J. Rösch à une grappe de raisin [4]. Le nombre de taches croît avec les dimensions du télescope utilisé, comme le montre la figure $4 a$ obtenue par A. Labeyrie au télescope de 5 mètres du Mont Palomar. Labeyrie [5] a identifié cette structure 
en grappe de raisin au phénomène de speckles couramment observé en lumière cohérente avec des lasers.

La relation de convolution (1) s'exprime plus simplement par transformation de Fourier comme un filtrage linéaire

$$
\widehat{I}(\mathbf{f})=\widehat{O}(\mathbf{f}) \cdot \hat{S}(\mathbf{f})
$$

où le symbole ${ }^{\wedge}$ représente une transformation de Fourier et $\mathbf{f}$ une fréquence spatiale (mesurée ici en $\left.\operatorname{radian}^{-1}\right)$. La connaissance de $S(\alpha)$ permet, en principe, de reconstituer l'objet par déconvolution dans le domaine de fréquences où $\widehat{S}(\mathbf{f}) \neq 0$. Malheureusement, si cette fonction peut quelquefois être mesurée en même temps que l'image [6], ce n'est guère possible dans le cas des observations astronomiques et il faut faire appel à des méthodes statistiques.

La fonction $S(\alpha)$ représente la figure de diffraction à l'infini d'une onde plane déformée par la turbulence traversant la pupille d'entrée du télescope. Si $\psi(\mathbf{r})$ est l'amplitude complexe aléatoire de cette onde déformée et $P(\mathbf{r})$ la transmission de la pupille (égale à l'unité à l'intérieur de la pupille et nulle à l'extérieur), $S(\alpha)$ est proportionnel au carré du module de la transformée de Fourier du produit $P(\mathbf{r}) \psi(\mathbf{r})$ [7]

$$
S(\boldsymbol{\alpha}) \propto|F(P(\mathbf{r}) \psi(\mathbf{r}))|^{2}
$$

où $\mathbf{r}$ est mesuré en unité de longueur d'onde. (Dans tout ce qui suit les longueurs seront mesurées en unité de longueur d'onde.)

Par transformation de Fourier, on obtient la fonction de transfert optique

$$
\widehat{S}(\mathbf{f}) \propto P(-\mathbf{r}) \psi(-\mathbf{r}) * \bar{P}(\mathbf{r}) \bar{\psi}(\mathbf{r})
$$

soit

$$
\widehat{S}(\mathbf{f}) \propto \int_{R^{2}} \bar{\psi}(\mathbf{r}) \psi(\mathbf{r}-\mathbf{f}) P(\mathbf{r}) P(\mathbf{r}-\mathbf{f}) \mathrm{d} \mathbf{r} .
$$

En l'absence de turbulence $\psi(\mathbf{r})=1$ (onde plane), d'où en normant la fonction de transfert à l'unité à l'origine :

$$
\widehat{S}(\mathbf{f})=\mathcal{S}^{-1} \cdot \int_{R^{2}} P(\mathbf{r}) P(\mathbf{r}-\mathbf{f}) \mathrm{d} \mathbf{r}=T(\mathbf{f})
$$

où $S$ est l'aire de la pupille. C'est l'expression bien connue de la fonction de transfert optique d'un instrument parfait limité par la diffraction [7].

1. Cas des images à long temps de pose. - La plupart des images astronomiques sont obtenues avec des temps de pose longs par rapport au temps d'évolution de l'image, de sorte que le résultat obtenu est assez bien représenté par la moyenne d'ensemble $\langle I(\alpha)\rangle$ donnée, compte tenu de l'éq. (1), par

$$
\left\langle I(\alpha)>={ }^{r} O(\alpha) *\langle S(\alpha)>\right.
$$

soit, par transformation de Fourier

$$
\langle\hat{I}(\mathbf{f})>=\hat{O}(\mathbf{f}) .\langle\hat{S}(\mathbf{f})>\text {. }
$$

La fonction de transfert pour les longues poses s'obtient donc en prenant la valeur moyenne de l'expression (4)

$$
\begin{aligned}
& <\hat{S}(\mathbf{f})>= \\
& \quad=s^{-1} \int\langle\bar{\psi}(\mathbf{r}) \psi(\mathbf{r}-\mathbf{f})>P(\mathbf{r}) P(\mathbf{r}-\mathbf{f}) \mathrm{d} \mathbf{r}
\end{aligned}
$$

$\psi(\mathbf{r})$ étant une fonction aléatoire stationnaire, le moment $\mathrm{du} 2^{\mathrm{e}}$ ordre

$$
C(\mathbf{f})=\langle\bar{\psi}(\mathbf{r}) \psi(\mathbf{r}-\mathbf{f})\rangle
$$

ne dépend que de $\mathbf{f}$ et la fonction de transfert optique moyenne s'écrit

$$
<\widehat{S}(\mathbf{f})>=C(\mathbf{f}) . T(\mathbf{f})
$$

produit de la fonction de transfert optique du télescope $T(\mathbf{f})$ par une fonction de transfert optique $C(\mathbf{f})$ décrivant l'effet de l'atmosphère.

La forme de la fonction $C(\mathbf{f})$ est maintenant bien connue théoriquement [8,9] et expérimentalement $[10,11]$. Lorsque le spectre des fluctuations d'indice de l'air suit la loi de Kolmogorov, elle est donnée par

$$
C(\mathbf{f})=\exp -3,44\left(f / f_{0}\right)^{5 / 3}
$$

où $f=|\mathbf{f}|$ et $f_{0}$ est un paramètre introduit par Fried [9] caractérisant l'état de la turbulence au moment de l'observation. C'est le diamètre du télescope qui, en l'absence de turbulence, donnerait la même résolution. Il est typiquement de l'ordre d'une dizaine de centimètres.

$f_{0}$ peut être, soit mesuré indépendamment de la prise de vue, soit estimé à partir de caractéristiques connues a priori dans l'objet observé [12]. La déconvolution est alors envisageable [12, 13, 14]. Malheureusement, la forme exponentielle de l'expression (11) montre que $\langle\hat{S}(\mathbf{f})\rangle$ décroît très rapidement avec la fréquence amenant $\langle\widehat{I}(\mathbf{f})\rangle$ en dessous du niveau de bruit du récepteur, ce qui rend en général peu efficace toute déconvolution.

2. Mesures interférométriques. - Michelson [15] et Anderson [16] ont montré les premiers la possibilité d'observer les hautes fréquences spatiales d'un objet astronomique en présence de turbulence atmosphérique. Leur méthode consiste à placer sur la pupille du télescope un écran percé de deux petites ouvertures décalées du vecteur f (Fig. 3). Lorsqu'on pointe le télescope sur une source ponctuelle, on observe dans le plan focal des franges d'Young. L'intensité sur l'axe est donnée par

$$
\begin{aligned}
& \quad|\psi(\mathbf{r})+\psi(\mathbf{r}-\mathbf{f})|^{2}= \\
& =|\psi(\mathbf{r})|^{2}+|\psi(\mathbf{r}-\mathbf{f})|^{2}+2 \operatorname{Re} \bar{\psi}(\mathbf{r}) \psi(\mathbf{r}-\mathbf{f}) .
\end{aligned}
$$




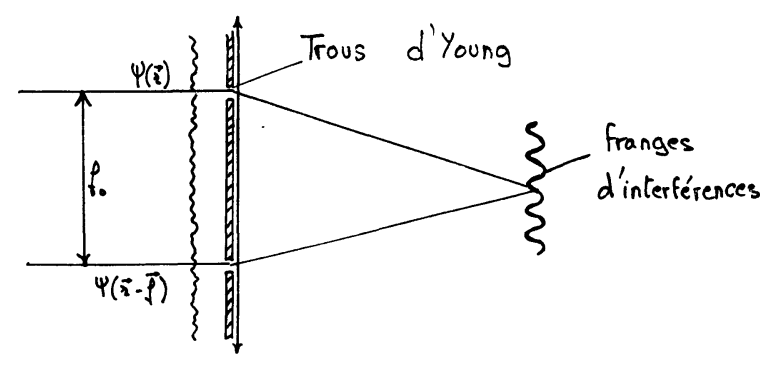

FIG. 3. - Principe de l'interféromètre stellaire de Michelson.

La quantité complexe $\bar{\psi}(\mathbf{r}) \psi(\mathbf{r}-\mathbf{f})$ représente le terme d'interférence. Son module donne la visibilité des franges et sa phase la position des franges. En présence de turbulence, ces quantités sont aléatoires.

On peut chercher à enregistrer photographiquement la figure d'interférence. Si le temps de pose est suffisamment long, les franges enregistrées seront caractérisées par la quantité

$$
\langle\bar{\psi}(\mathbf{r}) \psi(\mathbf{r}-\mathbf{f})>=C(\mathbf{f})
$$

et leur visibilité décroît avec la distance f des ouvertures comme la fonction $C(\mathbf{f})$. Pour un objet quelconque $O(\alpha)$, les franges sont caractérisées par le produit $\widehat{O}(\mathbf{f}) . C(\mathbf{f})$. Le résultat est l'équivalent à l'enregistrement d'une image à long temps de pose.

La supériorité des mesures de Michelson et Anderson provient du fait que leurs observations étaient visuelles. L'œil est en effet capable d'apprécier, au moins partiellement, la visibilité instantanée des franges. Plusieurs équipes essaient actuellement de reprendre ces observations en mesurant photoélectriquement la quantité

$$
\begin{aligned}
<|\bar{\psi}(\mathbf{r}) \psi(\mathbf{r}-\mathbf{f})|^{2}> & =\left\langle|\psi(\mathbf{r})|^{2}|\psi(\mathbf{r}-\mathbf{f})|^{2}>\right. \\
& =\langle J(\mathbf{r}) . J(\mathbf{r}-\mathbf{f})\rangle
\end{aligned}
$$

où $J(\mathbf{r})$ et $J(\mathbf{r}-\mathbf{f})$ sont les intensités lumineuses au niveau de chacune des deux ouvertures. Pour des séparations de plusieurs centimètres, ces deux intensités sont décorrélées et

$$
<|\bar{\psi}(\mathbf{r}) \psi(\mathbf{r}-\mathbf{f})|^{2}>=<J(\mathbf{r})>^{2}
$$

ce qui montre que la visibilité ainsi mesurée est de $100 \%$ quelle que soit la distance f. Pour un objet quelconque $O(\alpha)$, on obtient la quantité $|\widehat{O}(\mathbf{f})|^{2}$ sans aucune atténuation.

Les méthodes interférométriques ne permettent pas de mesurer commodément la phase de la quantité complexe $\hat{O}(\mathbf{f})$. On ne peut donc restaurer ainsi complètement l'objet $O(\alpha)$ mais seulement son autocorrélation.

3. «Speckle-interférométrie ». - Les mesures interférométriques montrent la supériorité des observations instantanées sur les enregistrements à long temps de

pose. De même, les images instantanées contiennent une information qui est perdue lors du passage à la valeur moyenne. La figure 4 montre, par exemple, des speckles enregistrés par A. Labeyrie au foyer du télescope de $5 \mathrm{~m}$ du Mont Palomar dans le cas d'une étoile non résolue par le télescope (Fig. $4 a$ ) et dans le cas d'une étoile résolue (Fig. $4 b$ ). Dans le deuxième cas, les speckles sont élargis par suite de la relation de convolution (1), information perdue en prenant la valeur moyenne car on fait alors disparaître les speckles. Il faut effectuer une analyse statistique qui préserve cette information.

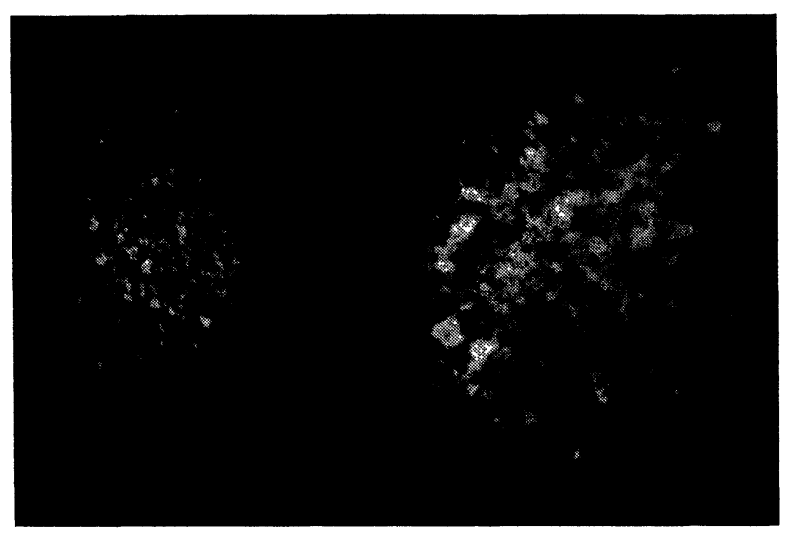

(a)

(b)

Fig. 4. - Speckles observés au télescope de $5 \mathrm{~m} \mathrm{du}$ Mont Palomar $(a)$ étoile non résolue, $(b)$ étoile résolue. (Clichés A. Labeyrie.)

La technique de speckle-interférométrie développée par A. Labeyrie [17] consiste à estimer la densité spectrale spatiale de l'image $\left\langle|\hat{I}(\mathbf{f})|^{2}\right\rangle$ par analyse statistique de quelques centaines de clichés à court temps de pose. Le calcul est fait analogiquement par compositage de figures de diffraction. La figure 5 montre des exemples de densités spectrales ainsi

(a)

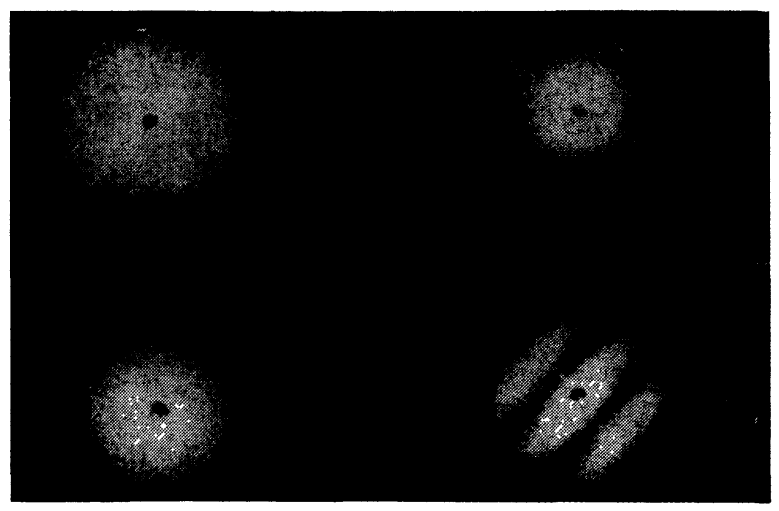

(b)

(d)

FIG. 5. - Densité spectrale des speckles produits par des étoiles de diamètre croissant $(a, b, c)$ et par une étoile double $(d)$. (Clichés A. Labeyrie.) 
obtenues. Dans le cas d'une étoile résolue, le diamètre de la figure de diffraction est inversement proportionnel au diamètre de l'étoile. Dans le cas d'une étoile double, on observe des franges mettant en évidence le dédoublement des speckles. L'interfrange est inversement proportionnel à la séparation des composantes. On peut séparer ainsi des composantes jusqu'à la limite de résolution théorique du télescope, malgré la turbulence.

En effet, l'éq. (2) conduit à :

$$
\left\langle|\widehat{I}(\mathbf{f})|^{2}\right\rangle=|\hat{O}(\mathbf{f})|^{2} \cdot\left\langle|\widehat{S}(\mathbf{f})|^{2}\right\rangle
$$

qui est à comparer à l'équation

$$
|<\widehat{I}(\mathbf{f})>|^{2}=|\hat{O}(\mathbf{f})|^{2} \cdot\left\langle\widehat{S}(\mathbf{f})>^{2}\right.
$$

déduite de (7) pour les images à long temps de pose. Tandis que la quantité $\langle\widehat{S}(\mathbf{f})\rangle^{2}$ décroît exponentiellement avec la fréquence (éq. (10) et (11)), la quantité $\left\langle|\hat{S}(\mathbf{f})|^{2}\right\rangle$ conserve des valeurs notables jusqu'à la fréquence de coupure du télescope. La figure 6

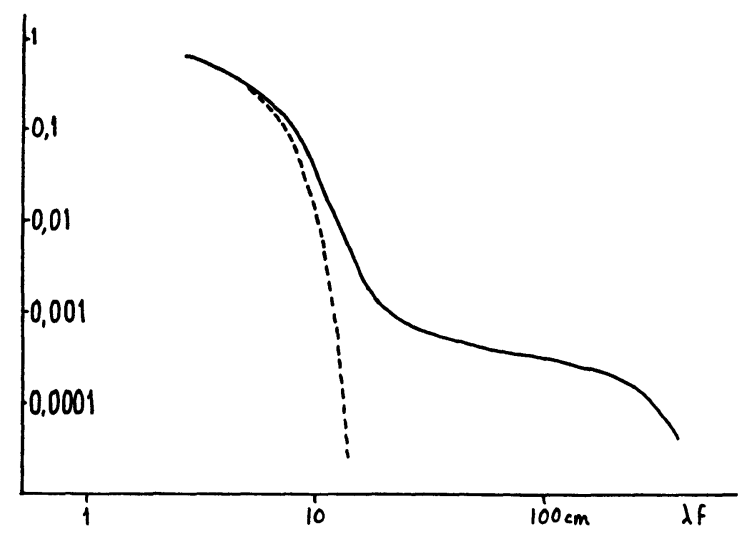

Fig. 6. - Densité spectrale des speckles produits par une source ponctuelle, calculée théoriquement par D. Korff (trait plein). La courbe en pointillé montre la densité spectrale théorique d'une image à long temps de pose.

montre l'allure théorique de cette fonction, pour le télescope de $5 \mathrm{~m}$ du Mont Palomar, calculée par Korff [1] à partir de l'éq. (4) et d'un modèle statistique de $\psi(\mathbf{r})$. La fonction $\left\langle|\hat{S}(\mathbf{f})|^{2}\right\rangle$ comporte un pic central et une aile haute fréquence. Sur les clichés de la figure 5 , le pic central très brillant a été artificiellement supprimé par un cache.

La théorie de la speckle-interférométrie [1, 2, 3] montre que l'aile haute fréquence de la fonction $<|\widehat{S}(\mathbf{f})|^{2}>$ est proportionnelle à la fonction de transfert optique $T(\mathbf{f})$ du télescope en l'absence de turbulence. Nous n'en donnerons ici qu'une démonstration heuristique en remarquant que la composante de Fourier de fréquence $f$ de l'image est une superposition des franges d'interférences produites par tous les couples de points de la pupille décalées du vecteur f. En l'absence de turbulence, ces différents systèmes de franges se superposent en phase. Si $N(\mathbf{f})$ est le nombre de ces couples de points

$$
\widehat{I}(\mathbf{f}) \propto N(\mathbf{f}) \hat{O}(\mathbf{f})
$$

soit en normant à l'unité la fonction de transfert à l'origine

$$
\widehat{I}(\mathbf{f})=\frac{N(\mathbf{f})}{N(O)} \hat{O}(\mathbf{f}) .
$$

L'expression de la fonction de transfert optique ainsi obtenue

$$
T(\mathbf{f})=N(\mathbf{f}) / N(O)
$$

est bien identique à (5) comme le montre la figure 7 .

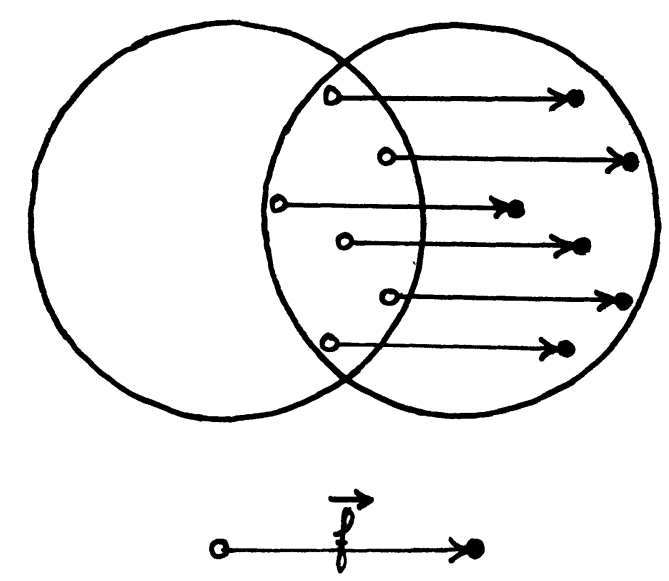

FIG. 7. - A l'intérieur d'un cercle, le nombre de couples de points décalés d'un vecteur $f$ est proportionnel à l'aire commune à deux cercles décalés de ce vecteur $\mathrm{f}$. Ce nombre détermine la fonction de transfert optique d'un télescope.

La turbulence a pour effet essentiel de déphaser aléatoirement les différents systèmes de franges d'une quantité $\varphi_{k}$ de sorte que

$$
\widehat{I}(\mathbf{f})=\frac{1}{N(O)} \widehat{O}(\mathbf{f}) \sum_{k=1}^{N(\mathbf{f})} \exp i \varphi_{k}
$$

d'où

$$
\left\langle|\widehat{I}(\mathbf{f})|^{2}>=\frac{1}{N^{2}(O)}|\hat{O}(\mathbf{f})|^{2}\left\langle\left|\sum_{k=1}^{N(\mathbf{f})} \exp i \varphi_{k}\right|^{2}\right\rangle\right.
$$

Pour des fréquences f suffisamment élevées, $\varphi_{k}$ est uniformément distribué entre 0 et $2 \pi$ et

$$
<\exp i \varphi_{k}>=0 \text {. }
$$

Les composantes haute fréquence ont complètement disparu dans les images à long temps de pose

$$
<\widehat{I}(\mathbf{f})>=\frac{1}{N(O)}(\mathbf{f}) \sum_{k=1}^{N(\mathbf{f})}<\exp i \varphi_{k}>=0 .
$$


Par contre, si $N(\mathbf{f})$ est pris maintenant égal au nombre de déphasages $\varphi_{k}$ statistiquement indépendants :

$$
\left.\left\langle\left|\sum_{k=1}^{N(\mathbf{f})} \exp i \varphi_{k}\right|^{2}\right\rangle=\sum_{k=1}^{N(\mathbf{f})}<\left|\exp i \varphi_{k}\right|^{2}\right\rangle=N(\mathbf{f})
$$

et la densité spectrale des images à court temps de pose est donnée par

$$
<|\widehat{I}(\mathbf{f})|^{2}>=|\hat{O}(\mathbf{f})|^{2} \frac{1}{N(O)} T(\mathbf{f}) .
$$

Elle est bien proportionnelle à la fonction de transfert optique du télescope avec cependant un facteur d'atténuation $1 / N(O)$ où $N(O)$ est le nombre de défauts statistiquement indépendants sur la surface d'onde. C'est aussi le nombre de speckles dans l'image. Ce facteur d'atténuation, inexistant dans le cas des mesures interférométriques, est dû à ce qu'une même composante de Fourier $\mathbf{f}$ est produite par interférence entre plusieurs couples de point de la pupille. La pupille est dite alors redondante. Goodman [18, 19] a développé l'utilisation de pupilles non redondantes permettant de supprimer cette atténuation.

4. Restauration d'images. - De même que les mesures interférométriques, la speckle-interférométrie conduit uniquement à une détermination de la densité spectrale $|\hat{O}(\mathbf{f})|^{2}$ de l'objet. La restauration complète de l'objet nécessite en outre la détermination de la phase de la quantité complexe $\hat{O}(\mathbf{f})$.

On peut considérer chaque speckle comme une image de l'objet. Par superposition d'un grand nombre de speckles brillants, Harvey et Lynds [20] semblent avoir obtenu une véritable image de disque stellaire. La méthode est cependant limitée à des objets angulairement très petits et l'erreur introduite par les speckles voisins est difficile à évaluer.

Un autre type de méthode est applicable lorsque l'objet comporte une source ponctuelle de référence

$$
O(\alpha)=\delta(\alpha)+O^{\prime}(\alpha)
$$

soit

$$
\widehat{O}(\mathbf{f})=1+\hat{O}^{\prime}(\mathbf{f})
$$

La densité spectrale obtenue par speckle-interférométrie

$$
|\widehat{O}(\mathbf{f})|^{2}=1+\hat{O}^{\prime}(\mathbf{f})+\overline{\hat{O}^{\prime}(\mathbf{f})}+\widehat{O}^{\prime}(\mathbf{f}) \overline{\hat{O}^{\prime}(\mathbf{f})}
$$

comporte alors le terme complexe cherché $\widehat{O}^{\prime}(\mathbf{f})$ que des techniques semblables à celle de l'holographie permettent d'isoler [21, 22]. Toutefois, il est exceptionnel de disposer d'une source ponctuelle de référence dans les limites de la région d'isoplanétisme et, si tel est le cas, une déconvolution directe est toujours possible [6] sans qu'il soit nécessaire de faire appel aux méthodes statistiques.
En fait, la phase de $\widehat{O}(\mathbf{f})$ peut être extraite statistiquement d'un grand nombre de clichés à court temps de pose sans qu'il soit nécessaire d'imposer aucune autre condition. L'éq. (2) s'écrit en effet en séparant module et phase

$$
\begin{aligned}
& |\hat{I}(\mathbf{f})| \exp i \varphi_{I}(\mathbf{f})= \\
& \quad=|\hat{O}(\mathbf{f})| \exp i \varphi_{0}(\mathbf{f}) \cdot|\widehat{S}(\mathbf{f})| \exp i \varphi_{S}(\mathbf{f})
\end{aligned}
$$

ce qui est équivalent aux deux équations

$$
\begin{aligned}
|\hat{I}(\mathbf{f})| & =|\widehat{O}(\mathbf{f})| \cdot|\hat{S}(\mathbf{f})| \\
\varphi_{I}(\mathbf{f}) & =\varphi_{0}(\mathbf{f})+\varphi_{S}(\mathbf{f})
\end{aligned}
$$

La phase $\varphi_{0}(\mathbf{f})$ de l'objet peut être déduite statistiquement de la phase $\varphi_{I}(\mathbf{f})$ de l'image en remarquant que les déphasages introduits par la turbulence sont en valeur moyenne nuls. La difficulté provient du fait que ces déphasages peuvent excéder largement $2 \pi$ alors que les phases $\varphi_{I}(\mathbf{f})$ obtenues par transformation de Fourier de $I(\alpha)$ sont comprises entre 0 et $2 \pi$. Il est donc nécessaire de déterminer la quantité

$$
\tilde{\varphi}_{I}(\mathbf{f})=\varphi_{I}(\mathbf{f})+2 k \pi
$$

par addition d'un multiple convenable de $2 \pi$. Ceci peut se faire par continuité à partir de la fréquence $O$ comme l'a montré Mc Glamery [23] dès 1969. On a alors

$$
\tilde{\varphi}_{o}(\mathbf{f})=\left\langle\tilde{\varphi}_{I}(\mathbf{f})\right\rangle
$$

Une autre méthode proposée récemment par Knox et Thompson [24] consiste à déterminer sur chaque image grad $\varphi_{I}$ au lieu de $\varphi_{I}$ avec un échantillonnage approprié. On a alors

$$
\tilde{\varphi}_{o}(\mathbf{f})=\int_{o}^{\mathbf{f}}<\operatorname{grad} \varphi_{I}>\mathrm{df} .
$$

Le résultat doit être le même quel que soit le chemin d'intégration.

Ces méthodes nécessitent le calcul de la transformée de Fourier bidimensionnelle $\widehat{I}(\mathbf{f})$ d'une centaine d'images $I(\alpha)$. Au lieu d'utiliser des images, nous avons proposé [25] d'utiliser des hologrammes donnant directement le module et la phase de $\hat{I}(\mathbf{f})$. Stroke et Reistrick [26] ont montré en 1965 la possibilité d'obtention d'hologrammes en lumière incohérente à l'aide d'un interféromètre à retournement de front d'onde. Ce type d'interféromètre a été développé en particulier par Mertz [27]. Il consiste à faire interférer l'onde incidente avec son symétrique par rapport à l'axe. La figure 8 montre le schéma d'un tel interféromètre.

Une étoile à la distance $\alpha$ de l'axe donne à travers l'interféromètre un système de franges d'intensité proportionnelle à

$$
1+\cos 4 \pi \boldsymbol{\alpha} \cdot \mathbf{r}
$$




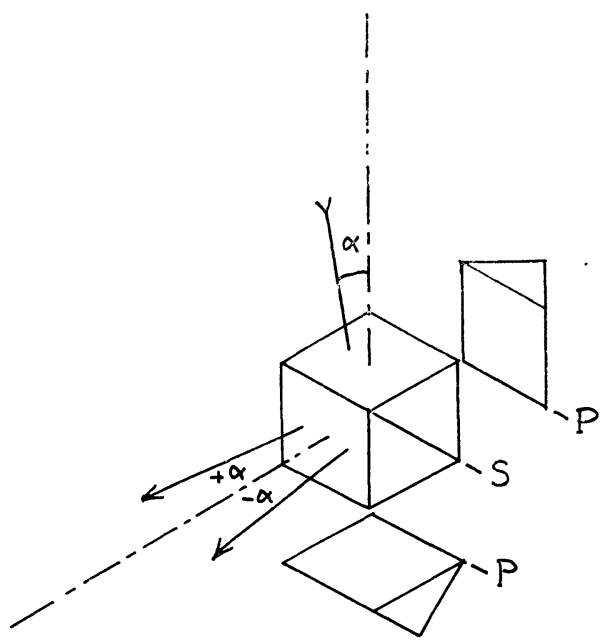

Fig. 8. - Principe d'un interféromètre à retournement de front d'onde. Un rayon faisant un angle $\alpha \neq 0$ avec l'axe donne naissance à deux rayons symétriques par rapport à l'axe optique. $\mathbf{P}$ : prisme en toit. $\mathbf{S}$ : séparatrice.

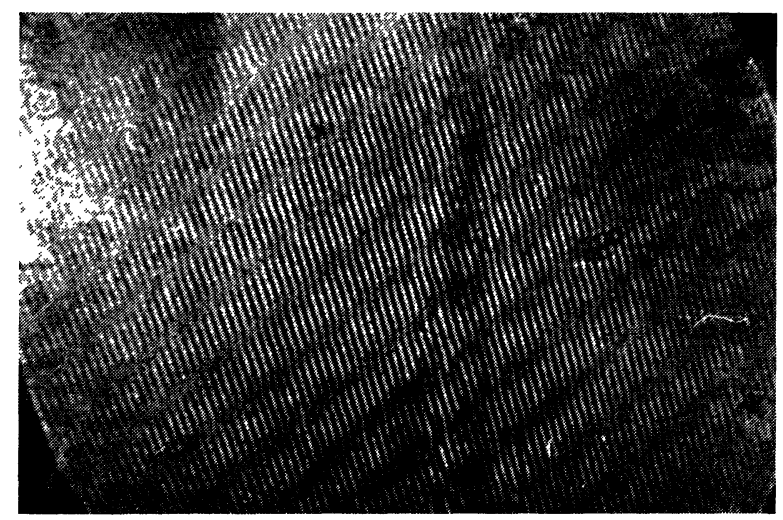

(a)

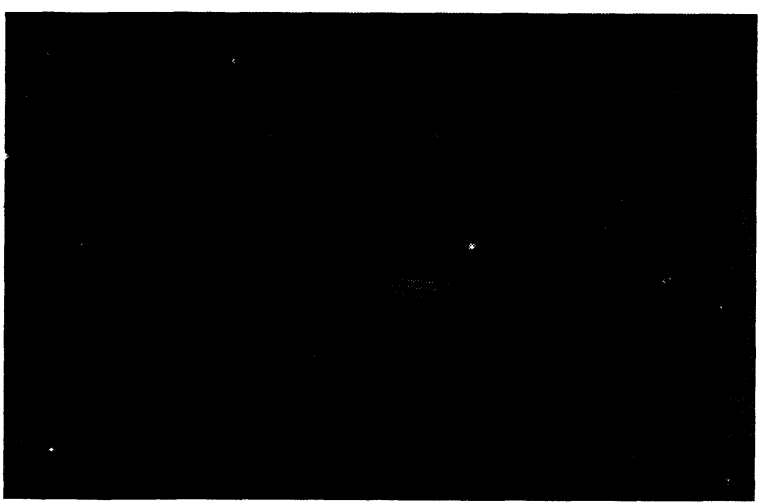

(b)

FIG. 9. - Hologrammes obtenus en lumière incohérente (bande passante $20 \AA$ ) des objets suivants; $(a)$ deux points lumineux simulant une étoile double, $(b)$ douze points lumineux dessinant une lettre R. (Clichés C. Froehly, A. Lacourt, C. Roddier.)

où $\mathbf{r}$ est, ici encore, mesuré en unité de longueur d'onde. Pour un objet incohérent de brillance $I(\alpha)$, les éclairements des systèmes de franges produits par chaque point de l'objet s'ajoutent et l'éclairement dans la figure d'interférence est donné par

$$
\begin{aligned}
\int I(\boldsymbol{\alpha}) \mathrm{d} \boldsymbol{\alpha}+\int I(\boldsymbol{\alpha}) \cos (4 \pi \boldsymbol{\alpha} \mathbf{r}) \cdot \mathrm{d} \boldsymbol{\alpha} & = \\
& =\widehat{I}(O)+\operatorname{Re} \hat{I}(2 \mathbf{r}) .
\end{aligned}
$$

Les figures $9 a$ et $b$ montrent des hologrammes de ce type obtenus par C. Froehly, G. Lacourt et C. Roddier.

Par transformation de Fourier inverse, on obtient

$$
\widehat{I}(O) \delta(\alpha)+\frac{1}{2}(I(\alpha)+I(-\alpha))
$$

ce qui redonne bien l'objet lorsque celui-ci est suffisamment hors de l'axe pour que $I(\alpha)$ et $I(-\alpha)$ ne se recouvrent pas. La figure 10 montre l'objet ainsi reconstitué en plaçant l'hologramme de la figure $9 b$ dans un faisceau laser.

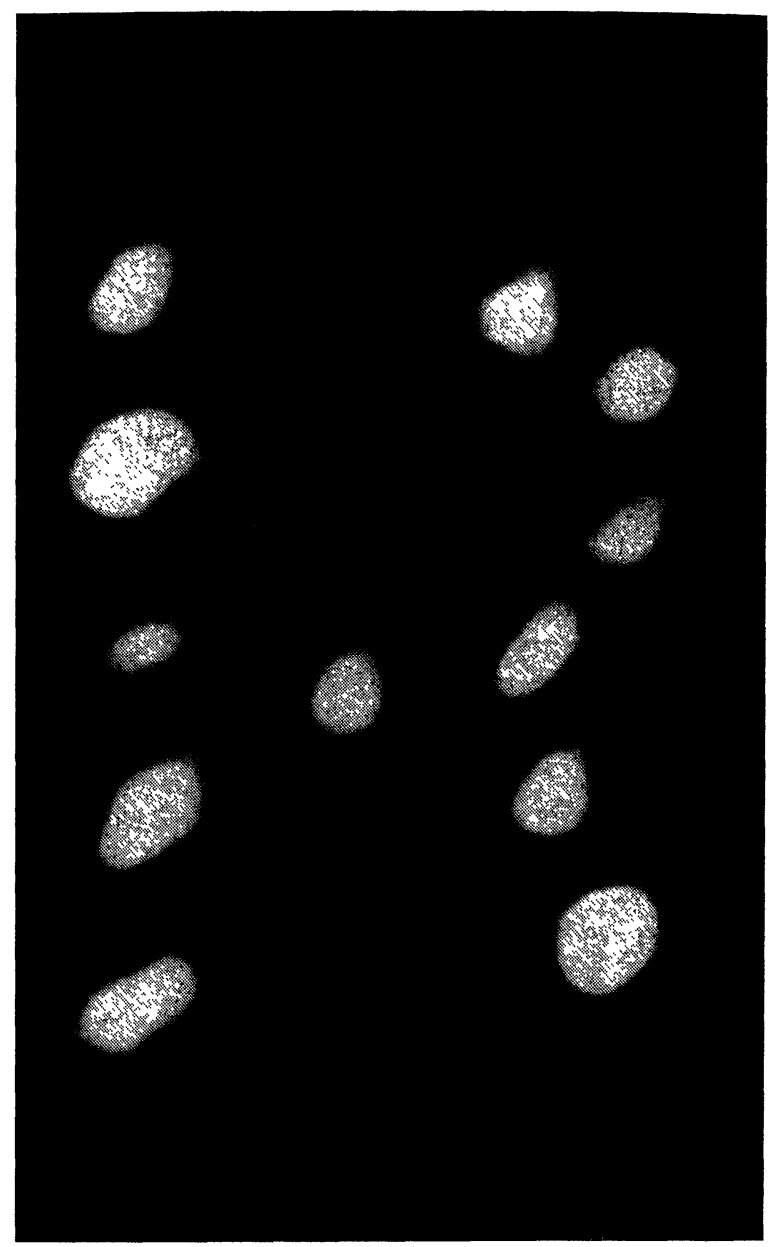

FIG. 10. - Reconstitution de l'objet obtenue en plaçant l'hologramme de la figure $9 b$ dans un faisceau laser.

Ce type d'interféromètre fonctionne comme un grand nombre d'interféromètres stellaires de Michelson en parallèle. En présence de turbulence, les franges d'interférence se déforment rapidement mais, du fait de l'absence de redondance, la visibilité des franges n'est 
pas affectée. On pourra, à l'aide d'un ordinateur, moyenner les phases lues sur un grand nombre d'hologrammes et reconstituer l'objet jusqu'à la fréquence de coupure du télescope par transformation de Fourier inverse. La figure 11 montre une reconstitution obtenue par transformation de Fourier bidimensionnelle numérique de l'hologramme de la figure $9 b$. Elle est à

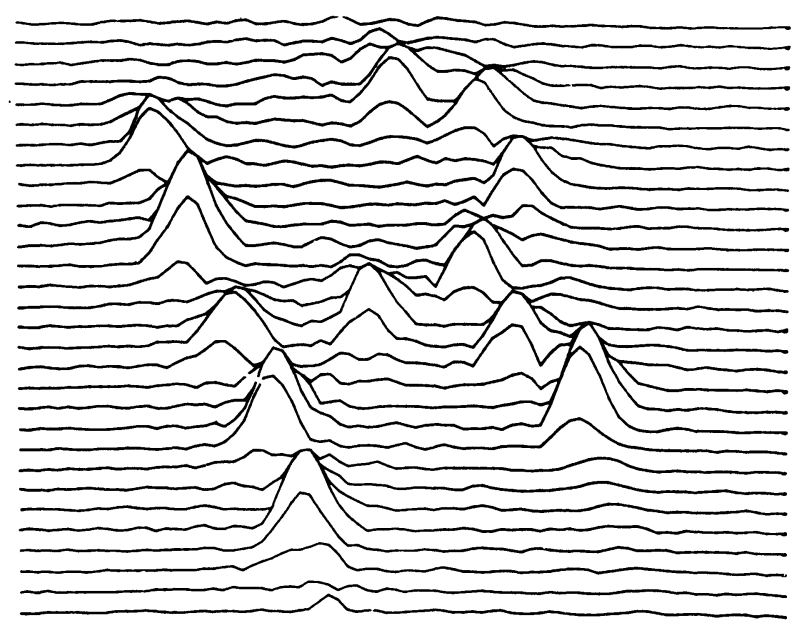

Fig. 11. - Reconstitution numérique de l'objet obtenue en analysant le cliché $9 b$ à l'aide d'un microdensitomètre PDS et en calculant la transformée de Fourier bidimensionnelle à l'aide d'un ordinateur PDP 11. (C. Roddier, Centre de dépouillement des clichés Astronomiques de l'I. N. A. G.) comparer avec la reconstitution analogique de la figure 10. Le programme de calcul de la phase moyenne est en cours d'élaboration (C. Roddier, non publié).

Des interféromètres de ce type ont déjà été utilisés pour l'étude des étoiles doubles ou des diamètres stellaires [28, 29, 30, 31, 32, 33]. Leur intérêt pour la restauration complète d'une image apparaît évident. Leur principal inconvénient est de nécessiter une bande passante étroite, inconvénient que l'on peut éliminer, au prix d'une complication du dispositif optique, en enregistrant simultanément la partie réelle et la partie imaginaire de la transformée de Fourier $\hat{I}(\mathbf{f})$ de l'image.

Enfin, plusieurs équipes aux Etats-Unis essaient actuellement de mesurer les déformations des surfaces d'ondes (interférométriquement ou avec des tests de Hartman) et de compenser ces déformations en temps réel à l'aide d'un miroir déformable. Ce sont des méthodes de restauration active par opposition aux méthodes passives décrites ci-dessus. Dans le cas des observations astronomiques, ces méthodes ont l'inconvénient de ne corriger que les défauts de phase sans tenir compte des fluctuations d'amplitude (scintillation) et, pour mesurer la correction à effectuer, de prélever une partie des photons disponibles dans un faisceau déjà par lui-même peu lumineux. La description détaillée de ces méthodes dépasserait le cadre de cet exposé.

\section{Bibliographie}

[1] Korff, D., J. Opt. Soc. Am. 63 (1973) 971.

[2] Dainty, J. C., Opt. Commun. 7 (1973) 129.

[3] Roddier, F., Opt. Commun. 10 (1974) 103.

[4] Rösch, J., C. R. Hebd. Séan. Acad. Sci. 247 (1958) 422.

[5] LABeYrie, A., Astron. and Astrophys. 6 (1970) 85.

[6] Mc Glamery, B. L., J. Opt. Soc. Am. 57 (1967) 293.

[7] Goodman, J. W., Introduction à l'optique de Fourier (Masson, Paris), 1972.

[8] Hufnagel, R. E. and Stanley, N. R., J. Opt. Soc. Am. 54 (1964) 52.

[9] Fried, D. L., J. Opt. Soc. Am. 56 (1966) 1372.

[10] Roddier, C. and Roddier, F., J. Opt. Soc. Am. 63 (1973) 661.

[11] Dainty, J. C. and Scaddan, R. J., Monthly Notices of the R. A. S. 170 (1975) 519.

[12] Coupinot, G., Icarus 19 (1973) 212.

[13] Harris, J. L., J. Opt. Soc. Am. 56 (1966) 569.

[14] Mueller, P. F. and Reynolds, G. O., J. Opt. Soc. Am. 57 (1967) 1338.

[15] Michelson, A. A., Astrophys. J. 51 (1920) 257.

[16] Anderson, J. A., Astrophys. J. 51 (1920) 263.

[17] Gezari, D. Y., Labeyrie, A. and Stachnik, R. V., Astrophys. J. 173 (1972) L 1.

[18] Russel, F. D. and Goodman, J. W., J. Opt. Soc. Am. 61 (1971) 182.

[19] Rhodes, W. T. and Goodman, J. W., J. Opt. Soc. Am. 63 (1973) 647
[20] Harvey, J., Lynds, R. and Worden, P., Cliché publié dans Scientific American 232 (1975) $n^{\circ} 2,42$.

[21] Liv, Y. C. and Lohmann, A. W., Opt. Commun. 8 (1973) 372.

[22] Gough, P. T. and Bates, R. H. T., Optica Acta 21 (1974) 243.

[23] Mc Glamery, B. L., Report NASA SP-256, 1969.

[24] Knox, K. T. and Thompson, B. J., Astrophys. J. 193 (1974) L 45.

[25] RodDIER, F., Recueil de séminaires (année 1973-74) (Observatoire de Besançon) p. 9.

[26] Stroke, G. W., An Introduction to Coherent Optics and Holography ( $2^{\mathrm{e}}$ édition, Academic Press, N. Y.), 1969, p. 158.

[27] MerTZ, L., Transformations in Optics (J. Wiley, N. Y.), 1965, chap. 4.

[28] Saunders, J. B., Astronom. J. 69 (1964) 449.

[29] Saunders, J. B., Precision Measurements, Advanced Optical Techniques, Van Heel edit. (North Holland), 1967 , p. 20.

[30] Kenknight, C. E., Astrophys. J. 176 (1972) L 43.

[31] Currie, D. G., Knapp, S. L. and Liewer, K. M., Astrophys. J. 187 (1974) 131.

[32] Breckinridge, J. B., Appl. Opt. 11 (1972) 2996.

[33] Breckinridge, J. B., Appl. Opt. 13 (1974) 2760. 